\title{
DIAGNÓSTICO DE TUMORES DO ÂNGULO PONTO-CEREBELAR COM O AUXÍLIO DE TÉCNICAS DE INTELIGÊNCIA ARTIFICIAL
}

\author{
FLÁVIO LEITÃO* FERNANDO C. GOMES ${ }^{* *}$ SEBASTIÃO DIÓGENES ${ }^{* * *}$, FLÁVIO LEITÃO FILHO****
}

\begin{abstract}
RESUMO - Trata-se de estudo multidisciplinar, cujo objetivo é a obtenção de modelo discriminatório entre diagnóstico de tumores do ângulo ponto-cerebelar (APC) e de distúrbios otorrinolaringológicos. Presentemente, a realização de um acurado exame neurológico e/ou otorrinolaringológico é incapaz de firmar diagnóstico de tumor do APC, sem valer-se de exames radiológicos de alto custo (tomografia computadorizada, ressonância magnética). O modelo proposto foi obtido através da utilização de técnicas de inteligência artificial e apresentou bom nível de acurácia $(88,4 \%)$ no teste de novos casos, considerando-se apenas o exame clínico e sem o auxílio de exames radiológicos.
\end{abstract}

PALAVRAS-CHAVE: tumor do ângulo ponto-cerebelar, distúrbio otorrinolaringológico, inteligência artificial, aprendizagem automática, indução em árvores de decisão.

\section{A diagnostic model for cerebellum-pontine angle tumors using artificial intelligence techniques}

ABSTRACT- We are concerned in this paper with learning classification procedures from known cases. More precisely, we provide a diagnostic model that discriminate between cerebellum-pontine angle (CPA) tumors and otorhinolaryngological (ENT) disorders. Usually, in order to distinguish between CPA tumors and ENT disorders one must perform clinical-neurological examination together with expensive radiological imagery (CT and MRI). The proposed model was obtained through artificial intelligence methods and presented a good accuracy level $(88.4 \%)$ when tested against new cases, considering only clinical examination without radiological imagery results.

KEY WORDS: cerebello-pontine angle tumor, otorhinolaryngological disorder, artificial intelligence, machine learning, decision tree induction.

Por ângulo pontocerebelar (APC), entende-se região da fossa posterior circundada pelas cisternas do cerebelo e pelo clivo e a face posterior do osso petroso, ventralmente ${ }^{1}$. Nessa região, o neurinoma ${ }^{2-6}$ do acústico e o meningioma ${ }^{7-9}$ são tumores por demais comuns nas casuísticas dos diversos serviços de todo o mundo. Entretanto, com menos frequência, outros tumores assentam-se no APC, dentre os quais: o lipoma ${ }^{10}$, o adenoma do saco endolinfático ${ }^{11}$, o carcinoma de células basais ${ }^{12}$, o cisto aracnóideo ${ }^{13}$, o epidermóide, o neurinoma do facial, o papiloma do plexo coroide, a metástase de neuroblastoma, o colesteatoma, o angioleiomioma, o hemangioma venoso, o angioma cavernoso $^{14}$, o glioma pontino ${ }^{15}$, o neurinoma dos nervos glossofaríngeo, vago e accessório ${ }^{16}$, o meduloblastoma $^{17}$, o ependimoma ${ }^{18}$, o melanoma ${ }^{19}$ e a neurilemomatose ${ }^{20}$. Além desses tumores, doenças parasitárias também ocorrem no APC como o cisto cisticercótico ${ }^{21}$. A presença de vasos

* Neurocirurgião, Professor do Departamento de Cirurgia da Faculdade de Medicina da Universidade Federal do Ceará (UFC); ** PhD, Pesquisador junto ao Laboratório de Inteligência Artificial (LIA) do Departamento de Computação da UFC, Bolsista Prod. em Pesquisa - CNPq. (carvalho@lia.ufc.br); *** Doutor, Professor do Departamento de Cirurgia da Faculdade de Medicina da UFC; ****Médico, Residente em Neurocirurgia, serviço do Prof. M. Samii, Hannover, Alemanha. Aceite: 16-novembro-1999.

Dr. Flávio Leitão - Rua Filadélfia 1390 - 60811-120 Fortaleza CE - Brasil. FAX 085244 6203. E-mail: flavioleitao@secrel.com.br 
anômalos, como no caso de alça vascular ${ }^{22}$ também é patologia encontradiça no APC. E, finalmente, processos infecciosos como o empiema ${ }^{23}$ são descritos como parte da coorte de patologias que se localizam no APC. Muito embora o neurinoma do acústico represente até $90 \%$ dos tumores do $\mathrm{APC}^{15}$, os meningiomas 3 a $5 \%{ }^{24}$, a existência de tão grande variedade de patologias dessa região, indiscutivelmente dificulta o diagnóstico. Tumores do APC apresentam sinais e sintomas que são decorrentes, principalmente, do comprometimento de estruturas ali localizadas como, por exemplo, dos nervos cranianos caudais. Tal comprometimento é multivariado e, nos casos de tumores, depende da rapidez de crescimento, da localização, da histologia, entre outros. Referidos sinais e sintomas não são patognomônicos de tumores do APC. Dessa forma, uma paralisia do IX nervo craniano, tanto poderia ser decorrência da existência de processo expansivo no APC, como simplesmente um processo degenerativo (e.g. esclerose em placa) ou mesmo um processo tóxico-infeccioso agudo (e.g. difteria). Como em muitos outros departamentos da economia humana, a certeza diagnóstica perseguida pelo médico algumas vezes não é alcançada, mormente quando da impossibilidade do uso de métodos auxiliares sofisticados e de alto custo, como tomografia computadorizada (TC) e ressonância magnética (RNM). Ademais, alguns sintomas associados aos tumores do APC são comuns a várias especialidades, de forma mais enfática à neurologia, à neurocirurgia e à otorrinolaringologia (ORL).

As informações sobre patologias do sistema nervoso avolumam-se nos bancos de dados dos hospitais do mundo inteiro. Sabe-se que a análise desses dados encontra-se dificultada por vários fatores, dentre os quais a inexistência de uma linguagem internacional única para descrevê-los. Muito pelo contrário, a esse aspecto junta-se o fato de referidos dados pecarem por: a) serem incompletos (ausência de alguns parâmetros necessários à formulação do diagnóstico); b) serem incorretos (avaliação incorreta ou subjetiva por parte do profissional responsável pelo exame do paciente); c) em alguns casos, serem esparsos (pequeno numero de casos ou prontuários com anotações pobres). Por esses fatos, métodos computacionais vêm sendo desenvolvidos para minorar essas deficiências ${ }^{25}$. O uso de sistemas computacionais em medicina vem sendo verificado há muito. Por exemplo, em neurocirurgia cita-se a TC e, mais recentemente, a neuronavegação ${ }^{26}$. Neste estudo, serão usadas técnicas computacionais para prover elementos de auxílio diagnóstico de tumores do APC.

Desde os primeiros dias da ciência da computação, um grande número de paradigmas computacionais surgiu para o estudo da inteligência artificial (IA). Os problemas de interesse da IA são, em geral, ligados a características inerentes ao homem. Por exemplo, ver e reconhecer formas, o processamento da linguagem natural, o raciocínio, o aprendizado automático, a robótica, etc ${ }^{27,28}$. Todas essas características são, em termos computacionais, muito difíceis. Isso significa que os computadores atuais, mesmo os supercomputadores, não conseguiriam dar uma resposta precisa dentro de um tempo aceitável, i.e. a proibitiva complexidade da entrada de dados geraria um processamento de anos, ou até séculos. Por isso, para resolvê-los, necessita-se de métodos especiais que conseguem evitar a complexidade dos problemas e encontrar uma resposta aproximada dentro de um tempo aceitável, usando-se os computadores disponíveis atualmente. Portanto, esses métodos computacionais de IA fazem com que a falha supracitada na descrição dos dados, não se constitua empecilho à aplicação da IA. O mesmo não se pode dizer dos métodos estatísticos paramétricos, correntemente usados em análise de dados.

Métodos de IA vêm sendo usados em vários ramos da atividade médica, notadamente através do uso de sistemas especialistas dedutivos e da indução de modelos de conhecimento a partir de casos, dentre outros. Para evidenciar o uso disseminado desses métodos em medicina, cita-se a sua aplicação na área cardíaca ${ }^{29}$, em oncologia ${ }^{30,31}$, em neuroanatomia ${ }^{32}$, em reprodução humana ${ }^{33}$, em traumatologia ${ }^{34}$, em reumatologia ${ }^{35}$, em neurologia $\mathrm{a}^{36}$, em imunologia $\mathrm{a}^{37} \mathrm{e}$ em oftalmologia ${ }^{38}$, entre outras.

O objetivo do estudo aqui apresentado é a obtenção de modelo de diagnóstico que seja capaz de diferenciar casos de tumores do APC dos casos de ORL, usando métodos indutivos de IA. Para alcançar o objetivo proposto, parte-se de casos pré-diagnósticados por serviços de neurocirurgia, bem como da clínica de ORL, como a seguir relatado. 


\section{CASUÍSTICA}

Os dados usados neste estudo advêm de três fontes: do serviço do Prof. Madjid Samii em Hannover, Alemanha; do Instituto de Neurocirurgia de Fortaleza; e da Clínica do Prof. Sebastião Diógenes. Eles perfazem um total de 750 casos. Desse total, 275 casos dizem respeito a tumores do APC (TAPC) e 475 casos representam relatos de distúrbios otorrinolaringológicos (DORL) que apresentavam sintomas encontradiços em casos de TAPC. A idade média dos pacientes é 50,49 anos. Os pacientes do sexo masculino perfazem 292, enquanto que os do sexo feminino totalizam 454. Em 4 casos o sexo não foi registrado.

A Tabela 1 apresenta as variáveis que descrevem a casuística. A coluna à esquerda relaciona as variáveis com os seus respectivos valores, a coluna à direita indica as siglas e códigos usados como entrada no banco de dados. As variáveis que podem assumir os valores "presença" ou "ausência", foram coletadas da seguinte forma: no prontuário do paciente, quando confirma-se a presença de certo sinal ou sintoma, este terá valor "presença", em contrapartida, a não citação indica o valor "ausência". Vale ressaltar que nenhuma das variáveis descritas exige o resultado de exames complementares.

\section{MÉTODO}

Dado um conjunto de casos (ou exemplos) conhecidos pelo diagnóstico confirmado, o objetivo é obter um modelo (em linguagem computacional: um classificador) a partir desses exemplos. $\mathrm{O}$ modelo obtido deverá ser capaz de diagnosticar, corretamente, casos idênticos aos usados para gerá-

Tabela 1. Variáveis que representam um caso e sua representação no banco de dados.

\begin{tabular}{|c|c|}
\hline Variáveis & Representação \\
\hline 1. Idade [em anos] & ID [idade] \\
\hline 2. Sexo [Feminino,Masculino] & $\mathrm{SX}[\mathrm{F}, \mathrm{M}]$ \\
\hline 3. Início da queixa [em meses] & IQ[meses] \\
\hline 4. Zumbido, acúfenus, tinitus [Presença, Ausência] & $\mathrm{Z} 8[\mathrm{P}, \mathrm{A}]$ \\
\hline 5. Perda dos sentidos, lipotímia [Presença, Ausência] & $\mathrm{CO}[\mathrm{P}, \mathrm{A}]$ \\
\hline 6. Dor (ou anestesia) nos $2 / 3$ anteriores da língua, dente ou córnea [Presença, Ausência] & D5[P,A $]$ \\
\hline 7. Dor (ou anestesia) no 1/3 posterior da língua [Presença, Ausência] & $\mathrm{DV}[\mathrm{P}, \mathrm{A}]$ \\
\hline 8. Dor (ou anestesia) na face [Presença, Ausência] & $\mathrm{DF}[\mathrm{P}, \mathrm{A}]$ \\
\hline 9. Diminuição da audição(hipoacusia, anacusia) [Presença, Ausência] & $\mathrm{D} 8[\mathrm{P}, \mathrm{A}]$ \\
\hline 10. Dificuldade de deglutição [Presença, Ausência] & $\mathrm{DD}[\mathrm{P}, \mathrm{A}]$ \\
\hline 11. Emese [Presença, Ausência] & $\mathrm{EM}[\mathrm{P}, \mathrm{A}]$ \\
\hline 12. Ataxia, vertigo, Romberg, Fournier [Presença, Ausência] & $\mathrm{EQ}[\mathrm{P}, \mathrm{A}]$ \\
\hline 13. Cefaléia [Presença, Ausência] & $\mathrm{CE}[\mathrm{P}, \mathrm{A}]$ \\
\hline 14. Diplopia [Presença, Ausência] & $\mathrm{D} 6[\mathrm{P}, \mathrm{A}]$ \\
\hline 15. Paresia [Presença, Ausência] & $\mathrm{PA}[\mathrm{P}, \mathrm{A}]$ \\
\hline 16. Tontura [Presença, Ausência] & $\mathrm{TO}[\mathrm{P}, \mathrm{A}]$ \\
\hline 17. Sensibilidade gustativa diminuição nos $2 / 3$ anteriores [Presença, Ausência] & $\mathrm{SF}[\mathrm{P}, \mathrm{A}]$ \\
\hline 18. Sensibilidade gustativa diminuição no $1 / 3$ posterior [Presença, Ausência] & $\mathrm{S} 9[\mathrm{P}, \mathrm{A}]$ \\
\hline 19. Disartria [Presença, Ausência] & $\mathrm{DI}[\mathrm{P}, \mathrm{A}]$ \\
\hline 20. Nistagmus [Presença, Ausência] & $\mathrm{NI}[\mathrm{P}, \mathrm{A}]$ \\
\hline 21. Outros nervos [Presença, Ausência] & $\mathrm{OU}[\mathrm{P}, \mathrm{A}]$ \\
\hline 22. DIAGNÓSTICO [TAPC,DORL] & D[TAPC,DORL] \\
\hline
\end{tabular}


lo, assim como casos desconhecidos. Este tópico tem sido desenvolvido por pesquisadores de IA e chama-se aprendizagem automática (ou "machine learning").

Vale relembrar que os dados são descritos de forma imperfeita. Neste caso, é de se esperar que o modelo gerado seja também imperfeito, uma vez que ele pode ser afetado pelas irregularidades estatísticas presentes na descrição dos casos. Diz-se então que o modelo obtido excede ("overfit") o conjunto de casos. Isso significa que o modelo consegue diagnosticar corretamente quase todos os casos novos idênticos aos usados para gerá-lo, porém, é impreciso quando se trata de diagnosticar novos casos desconhecidos. Deste modo, deve-se buscar classificadores que sejam menos ligados à casuística disponível, porém, mais gerais, i.e. que consigam definir corretamente o diagnóstico de novos casos. Um modelo mais geral é menos complexo, no que diz respeito ao seu tamanho ("rightfit"). Em resumo, busca-se um bom compromisso entre complexidade e o número de diagnósticos corretos, considerando-se apenas os casos disponíveis que alimentaram o sistema.

O método utilizado consiste no aprendizado automático de árvores de decisão com complexidade limitada. Este método chama-se CART ("Classification and Regression Trees") e foi proposto por Breiman et al. ${ }^{39}$. CART foi escolhido por lidar elegante e eficientemente com o problema que consiste em encontrar modelos representados por árvores de decisão com complexidade limitada. Ademais, CART e outros métodos que se utilizam de árvores de decisão, têm sido usados com sucesso na geração de modelos de diagnóstico em medicina ${ }^{31,40}$.

A Figura 1 ilustra de forma didática uma árvore de decisão com dois nós(círculos), quatro ramos (V1-V4) e três folhas (retângulos). Árvores de decisão são estruturas que imitam uma árvore invertida, em que as folhas (retângulos) representam diagnósticos, os nós (círculos) representam sintomas e os ramos são os valores assumidos pelo sintoma (ou sinal) a ele associado. O primeiro nó é chamado de raiz.

Inicialmente CART gera uma árvore de decisão de tamanho máximo a partir da descrição da casuística, i.e. cada caminho que vai da raiz a uma folha, representa um único caso. Em seguida é efetuada uma poda (corte de ramos). A poda gerará uma sequência de árvores cada vez menores. Finalmente a melhor árvore é escolhida através de processos estatísticos. Maiores detalhes sobre CART podem ser obtidos em "Classification and Regression Trees" Breimen et al. ${ }^{39}$.

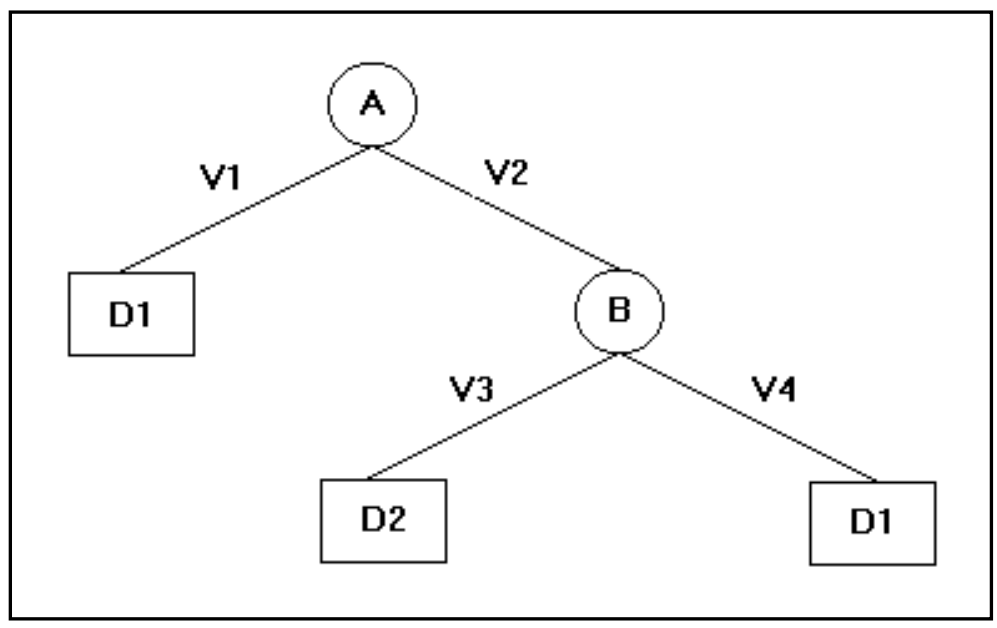

Fig 1. Árvore de decisão. Desse modelo podem-se extrair três regras de diagnóstico: se A $=V 1$ implica diagnóstico D1; se $A=V 2$ e $B=V 3$ implica D2; e se $A=V 2$ e $B=V 4$ implica diagnóstico D1. 
Tabela 2. Matriz de frequências dos casos de teste (RTAPC,RDORL) versus diagnóstico do modelo (HTAPC,HDORL).

\begin{tabular}{lcc}
\hline & RTAPC & RDORL \\
\hline HTAPC & PP & FP \\
HDORL & FN & NN \\
\hline
\end{tabular}

PP representa os casos de tumores do APC diagnosticados corretamente pelo modelo, $N N$ representa os casos de ORL corretamente diagnosticados pelo modelo, FN são os falsos negativos e FP os falsos positivos.
Tabela 3. Matriz de frequências obtidas na avaliação da árvore de decisão da Figura 2.

\begin{tabular}{lcc}
\hline & RTAPC & RDORL \\
\hline HTAPC & 212 & 63 \\
HDORL & 24 & 451 \\
\hline
\end{tabular}

A Tabela 2 apresenta a matriz de frequências, segundo o diagnóstico. O modelo final obtido será avaliado através da comparação entre o diagnóstico gerado pelo modelo (HTAPC,

HDORL) e o diagnóstico real de cada caso (RTAPC, RDORL), testado à luz de casos independentes. A acurácia do modelo será dada pela expressão $(P P+N N) /(R T A P C+R D O R L)$, i.e. o número de casos diagnosticados corretamente pelo modelo, dividido pelo número total de casos. Essa medida nos fornece o poder preditivo do modelo. A sensibilidade do modelo com relação aos casos de tumores do APC é dada pela fórmula $P P / R T A P C$. Portanto, a árvore de decisão obtida será avaliada segundo a acurácia e a sensibilidade.

\section{RESULTADOS E DISCUSSÃO}

A Figura 2 apresenta a árvore de decisão fornecida pelo CART. A variável que representa paresia (PA, vide Tabela 1) está localizada na raiz da árvore e apresenta, consequentemente, o maior poder discriminatório. Vale ressaltar que, dos 121 casos que apresentavam paresia, 117 foram diagnosticados corretamente pelo modelo. Para diagnosticar todos os casos o modelo gerado, além de PA se utiliza de apenas mais três variáveis descritas na Tabela 1, quais sejam: EQ, DF, TO.

Considerando a totalidade dos casos (750), o modelo diagnosticou corretamente 88,4\% (663) o que representa uma boa acurácia. A Tabela 3 mostra a matriz de frequências para a árvore da Figura 2. Separando-se apenas os casos de tumores do APC (275), nota-se que a árvore de decisão consegue acertar o diagnóstico de 212 casos $(77,09 \%)$. Além disso, o número de falsos negativos (FN) foi apenas 24 em 750, o que corresponde a 3,2\%.

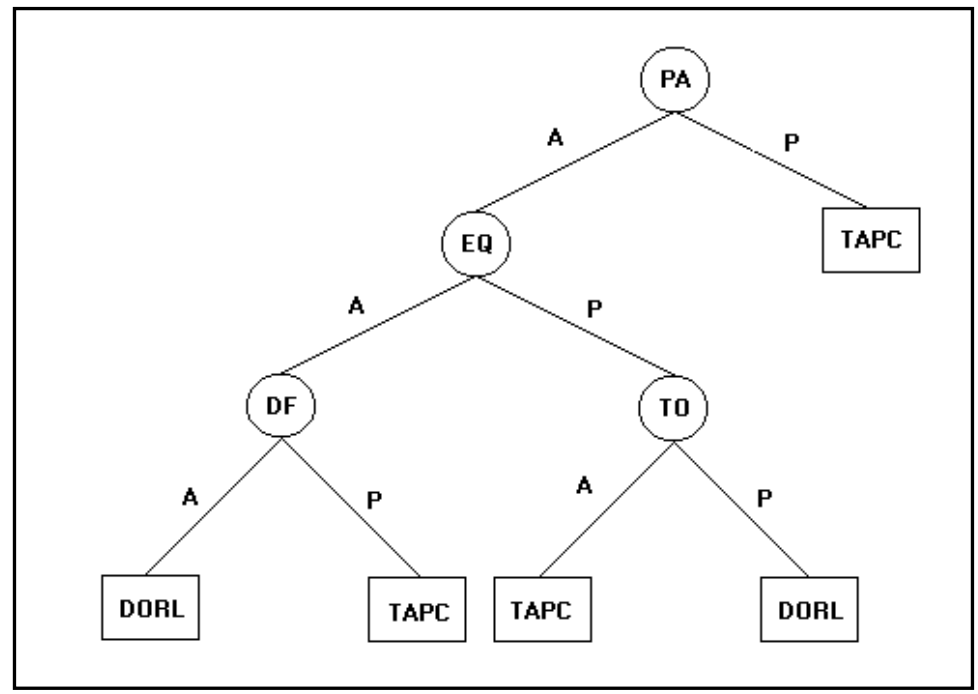

Fig 2. Árvore de decisão fornecida por CART 
Além da boa capacidade discriminatória, o modelo discriminatório proposto não envolve variáveis obtidas após exames complementares invasivos ou imagens. Em consequência, o modelo é de grande interesse como indicador do correto diagnóstico, notadamente quando da espera dos resultados de exames complementares ou na impossibilidade de realizá-los.

Os resultados aqui relatados são animadores. Eles demonstram que árvores de decisão de alta performance podem ser geradas a partir de dados que apresentam várias formas de ruídos, conforme discutido na introdução. Ademais, o fato de dispormos de um número maior de casos de ORL, não significou perda de acurácia da árvore de decisão gerada, com relação ao diagnóstico de tumores do APC. Planeja-se a realização de outros experimentos, utilizando-se o mesmo conjunto de dados. Pretendese criar modelo similar ao aqui apresentado para o diagnóstico histopatológico de tumores do APC.

Agradecimentos - O Professor Madjid Samii da Universidade de Hannover, cedeu gentilmente seus arquivos de casos relativos a tumores do APC, sem os quais não teria sido possível a realização deste estudo.

\section{REFERÊNCIAS}

1. Lang J. Clinical anatomy of the posterior cranial fossa and its foramina. Stuttgart: Georg Thieme Verlag, Stuttgart, 1991:83-84.

2. Ito K, Kurita H, Sugasawa K, Mizuno M, Sasaki T. Analysis of neuro-otological complications after radiosurgery for acoustic neurinomas. Int J Radiation Oncol Biol Phys 1977;39:983-988.

3. KuoTC, Jackler RK, Wong K, Blevins NH, Pitts LH. Are acoustic neuromas encapsulated tumors?. Otolaryngol Head Neck Surg 1997;117:606-609.

4. Monsell EM, Cody DD, Spickler E, Windham JP. Segmentation of acoustic neuroma with magnetic resonance imaging and eigen image filtring. Am J Otol 1997;18:602-607.

5. Noguchi Y, Komatsuzaki A, Yamada I, Okuno H, Haraguchi H. Radiology in focus vestibular schwannoma showing a dural tail on contrast-enhanced magnetic resonance images. J Laryngol Otol 1997;111:877-879.

6. Matthies C, Samii M. Direct brainstem recording of auditory evoked potentials during vestibular chivannoma resection: nuclear BAEP recording. Technical note and preliminary results. J Neurosurgery 1997;86:1057-1062.

7. Baguley DM, Beynon GJ, Grey PL, Hardy DG, Moffat DA. Audio-vestibular findings in meningioma of the cerebellopontine angle: a retrospedtive review. J Laryngol Otol 1997;3:1022-1026.

8. Weissman JL. Imaging of Meniere's disease. Otolaryngol Clin N Am 1997;30:1105-1116.

9. Zeitouni AG, Zagzag D, Cohen NL. Meningioma of the internal auditory canal. Ann Otol Rhinol Laryngol 1997;106:657-661.

10. Behar PM, Dolan R, Dastur K, Marrangoni AG, Nayak N. Fibrovascular lipoma of the cerebellopontine angle mimicking trigeminal neuralgia. ENT 1998;1:58-60.

11. Folker RJ, Meyerhoff WL, Rushing EJ. Aggressive papillary adenoma of the cerebellopontine angle: case report of an endolymphatic sac tumor. Am J Otolaryngol 1997;18:135-139.

12. Gall C, Buttner A, Bise K, Steiger H-J. Primary intracranial metatypical basal cell carcinoma: case report. Neurosurgery 1997;41:279-282.

13. Jallo GI, Woo HH, Meshki C, Epstein FJ, Wisoff JH. Arachnoid cysts of the cerebello pontine angle: diagnosis and surgery. Neurosurgery 1997;40:31-38.

14. Comey CH, Kondziolka D, Howard Y. Regional parenchymal enhancement with mixed cavernous/venous malformation of the brain: case report. J Neurosurg. 1997;86:154-158.

15. Kohan D, Downey LL, Lim J, Cohen NL Elowitz E. Uncommon lesions presenting as tumors of the internal auditory cannal and cerebello pontine angle. Am J Otol 1997;18:386-392.

16. Krauss JK Seeger W, Jankovic J. Cervical dystonia associated with tumours of the posterior fossa. Mov Disord 1997; 12:443-447.

17. Mehta JS, Sharr MM. Oncology in focus: an unusual cause of acute labyrithine failure. J Laryngol Otol 1998;112:193-195.

18. Sanford RA, Kun LE, Heideman RL, Gajjar A. Cerebellar pontine angle ependymoma in infants. Pediatr Neurosurg 1997;27:84-91

19. Shinogami M, Yamasoba T, Sasaki T. International case reports. Otolaryngol Head Neck Surg 1996;118:276- 279.

20. Shishiba T. Follow-up study of a patient with neurilemmomatosis. J Am Acad Dermatol 1997;37:797-799.

21. Del Brutto $\mathrm{OH}$. Albendazole therapy for subarachnoid cysticerci: clinical and neuroimaging analysis of 17 patients. $\mathbf{J}$ Neurol Neurosurg Psychiatry 1997;62:659-661.

22. Herzog JA, Bailey S, Meyer J. Vascular loopes of the internal auditory cannal: a diagnostic dilemma. Am J Otol 1997; 18:26-31

23. Nathoo N, Nadvi SS, van Dellen JR. Infratentorial empyema: analysis of 22 cases. Neurosurgery 1997;41:1263-1269.

24. Asawavichianginda S, Vaewvichit K, Taecholarn C. Cerebellopontine angle meningiomas with primary otologic symptoms. J M A of Thailand 1997;80:603-608.

25. Lavrac N. Selected techniques for data mining in medicine. Artif Intellig Med 1999;16: 3-23.

26. Meyer B, Schaller C, Van Roost D, Schramm J. Benefits and pitfalls of neuronavigation in neurosurgical routine: a critical summary after a consecutive series of 120 patients (Abstr). Hannover, 1998: 49th. Annual Meeting of German Society of Neurosurgery.

27. Benabid AL, Koudsie A, Ashraf A, Hoffmann D, Kebas JF. Robotics in neurosurgery (Abstr). Hannover, 1998: 49th. Annual Meeting of German Society of Neurosurgery. 
28. Rabischong P. Present and future of surgical robotics: a robionic concept (Abstr.). Hannover, 1998: 49th. Annual Meeting of Germany Society of Neurosurgery.

29. Komorowski J, Øhrn A. Modelling prognostic power of cardiac tests using rough sets. Artif Intellig Med 1999;15: 167-191.

30. Fogel DB, Wasson III EC, Boughton EM, Porto VW. Evolving artificial neural networks for screening features from mammograms. Artif Intellig Med 1998;14: 317-326.

31. Carvalho-Gomes F, Gascuel O. SDL, a stochastic algorithm for learning decision lists with limited complexity. Ann Mathemat Artif Intellig 1994;10:281-302.

32. Gibaud B, Garlatti S, Barillot C, Faure E. Computerized brain atlases as decision support systems: a methodological approach. Artif Intellig Med 1998;14, 83-100.

33. Jurisica I, Mylopoulos J, Glasgow J, Shapiro H, Casper RF. Case-based reasoning in IVF: prediction and knowledge mining. Artif Intellig Med 1998;12:1-24.

34. Innocent PR, Barnes M, John R. Application of fuzzy ART/MAP and MinMax/MAP neural network models to radiographic image classification. Artif Intellig Med 1997;11:241-263.

35. Zupan B, Dzeroski S. Acquiring background knowledge for machine learning using function decomposition: a case study in reumatology. Artif Intellig Med 1998;14:101-117.

36. Toffano-Nioche C, Beroule D, Tassin JP. A functional model some Parkinson's disease symptoms using a guided propagation network. Artif Intellig Med 1998;14:237-258.

37. Lathrop RH, Steffen NR, Raphael MP, et al. Knowledge-based avoidance of drug-resistant HIV mutants. AI Magazine 1999;20:13-25.

38. Poggio T, Beymwe D. Learning to see. IEEE Spectrum 1996; May:60-69.

39. Breiman L, Friedman JH., Olshen RA, Stone CJ. Classification and regression trees. Belmont: Wadsworth, 1984.

40. Quinlan, JR, Compton, PJ, Horn, KA, Lazarus, L. Inductive knowledge acquisition: a case study. In Quinlan JR (ed) Application of expert systems. Reading: Addison Wesley, 1987:157-173. 